\title{
THE IMPACT OF THE CUSHING'S DISEASE REMISSION IN ASSOCIATED \\ COMORBIDITIES
}

$\underline{V e r a ~ F e r n a n d e s ~}^{1}$, Maria Joana Santos ${ }^{1}$, Rui Almeida ${ }^{2,3}$, Olinda Marques ${ }^{1,3}$

${ }^{1}$ Department of Endocrinology, ${ }^{2}$ Department of Neurosurgery, ${ }^{3}$ Pituitary Tumours Group, Hospital of Braga, Portugal

\section{BACKGROUND}

The Cushing's disease (CD) is associated with comorbidities that have a significant impact on patients' quality of life. However, it is not known the true impact of the disease remission on these comorbidities.

We aim to assess the evolution of comorbidities associated with $C D$, after its remission.

\section{RESULJS}

\section{$>77.8 \%$ i $\mid 22.2 \%$}

$>$ Mean Age: $\mathbf{3 9 . 6 \pm 1 5 . 1}$ years[14-69]

$>1$ pediatric patient ( 14 years)

$>$ Treatment before remission: 17 patients (94.4\%) were in remission after

surgery and $1(5.6 \%)$ required adjuvant radiotherapy

$>$ Time of follow-up: Mean 4.2 years [2.2-5.4]

\section{COMORBIDITIES AT DIAGNOSIS}

At diagnosis, 12 patients had hypertension, 3 had type 2 diabetes (T2DM), 6 dyslipidemia and 7 were on psychotropic drugs.

\begin{tabular}{|c|c|c|c|}
\hline \multirow{2}{*}{\multicolumn{2}{|c|}{ Anti-hipertensive drugs }} & At diagnosis & After remission \\
\hline & 0 & $6(33.3 \%)$ & $14(77.8 \%)$ \\
\hline \multirow{6}{*}{ 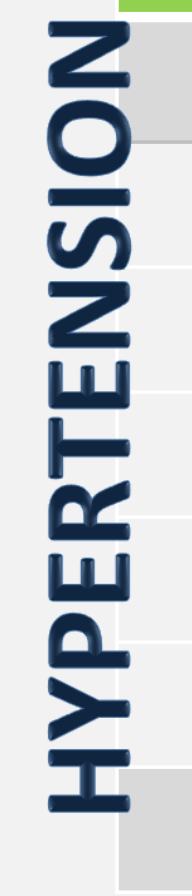 } & 1 & $5(27.8 \%)$ & $3(16.7 \%)$ \\
\hline & 2 & $1(5.6 \%)$ & \\
\hline & 3 & $3(16.7 \%)$ & $1(5.6 \%)$ \\
\hline & 4 & $2(11.1 \%)$ & \\
\hline & 7 & $1(5.6 \%)$ & - \\
\hline & TOTAL (with drugs) & $12(66.7 \%)$ & $4(22.2 \%)$ \\
\hline & ral antibiabetic drugs & At diagnosis & After remission \\
\hline & 0 & $15(83.3 \%)$ & $15(83.3 \%)$ \\
\hline & 1 & $1(5.6 \%)$ & $2(11.1 \%)$ \\
\hline 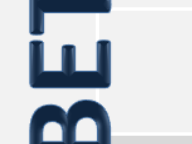 & 2 & $2(11.1 \%)$ & $1(5.6 \%)$ \\
\hline $\bar{\varangle}$ & TOTAL (with drugs) & $3(16.7 \%)$ & $3(16.7 \%)$ \\
\hline & Insulinotherapy & At diagnosis & After remission \\
\hline & 0 & $17(94.4 \%)$ & $18(100 \%)$ \\
\hline & & $1(5.6 \%)$ & \\
\hline
\end{tabular}
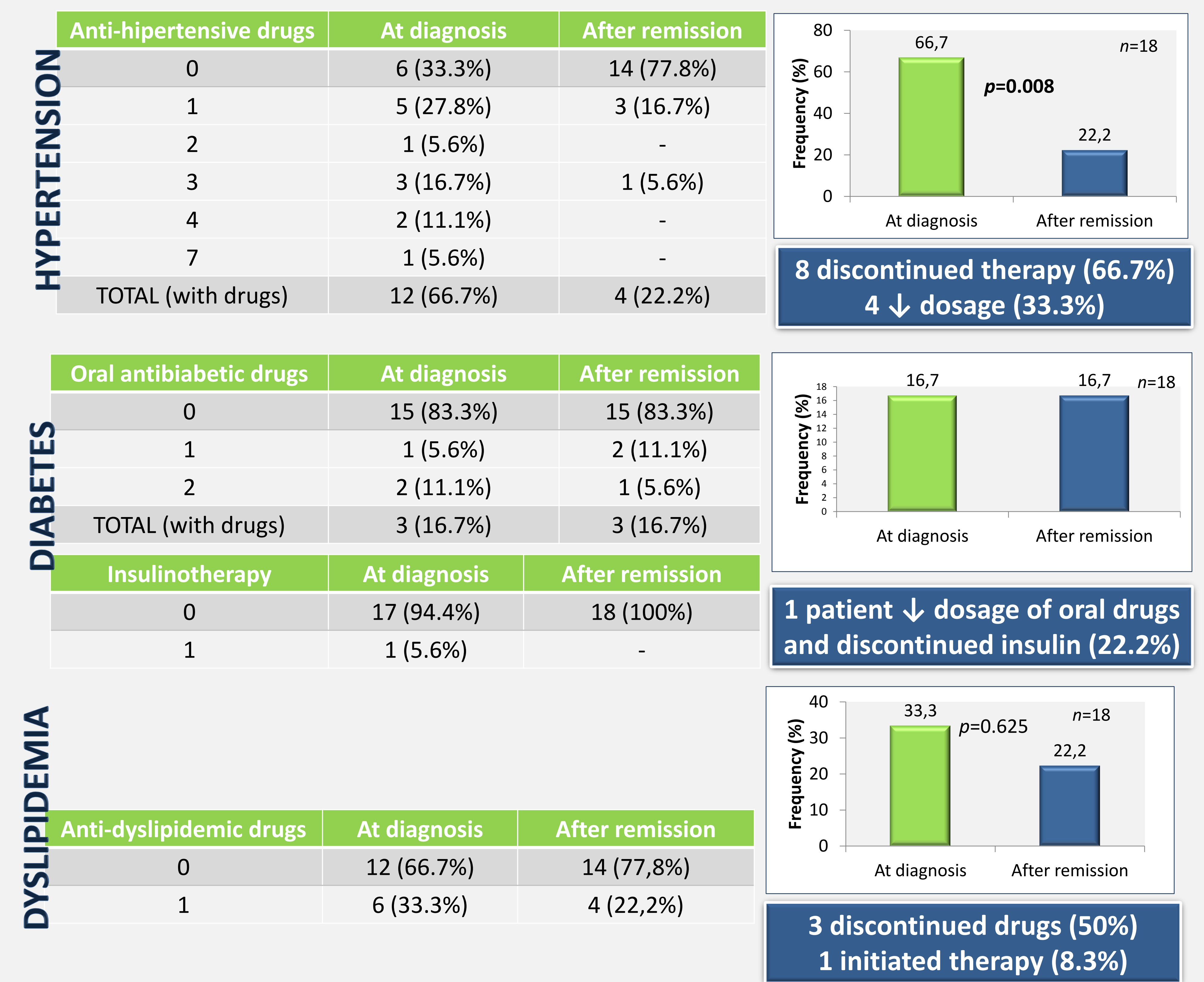

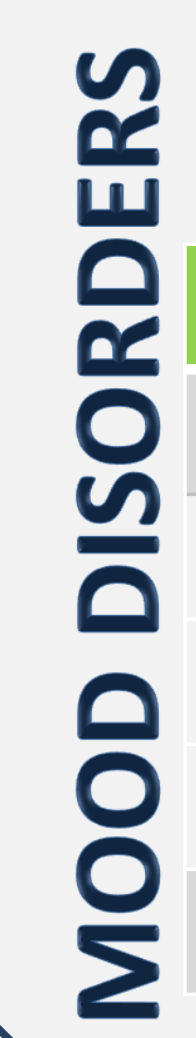

\begin{tabular}{|c|c|c|}
\hline sychotropic drugs & At diagnosis & After remission \\
\hline 0 & $11(61.1 \%)$ & $15(83.3 \%)$ \\
\hline 1 & $3(16.7 \%)$ & $1(5.6 \%)$ \\
2 & $2(11.1 \%)$ & $1(5.6 \%)$ \\
3 & $2(11.1 \%)$ & $1(5.6 \%)$ \\
\hline TOTAL (with drugs) & $7(38.9 \%)$ & $3(16.7 \%)$ \\
\hline
\end{tabular}

\section{METHODS}

- Observational, analytical and retrospective study

18 patients with $C D$ in remission, followed in our Department

Clinical data concerning the time of diagnosis (active disease) and one year after surgery/radiotherapy (remission) were collected

\section{Statistical Analysis:}

IBM ${ }^{\circledR}$ SPSS $^{\circledR}$ Statistics v. $20.0 \mid p<0.05 \rightarrow$ statistically significant

\section{OSTEOPOROSIS/OSTEOPENIA}

Eleven patients (61.1\%) underwent initial dual-energy $\mathrm{x}$-ray absorptiometry, 2 had osteoporosis and 4 osteopenia

\begin{tabular}{|c|c|c|}
\hline Diagnosis & At diagnosis & After remission \\
\hline Osteoporosis & $2(18.2 \%)$ & 0 \\
\hline Osteopenia & $4(36.4 \%)$ & $\begin{array}{c}1 \text { osteopenia }(9.1 \%) \\
1 \text { Normal } \\
2 \text { await reavaluation }\end{array}$ \\
\hline TOTAL & $6(54.5 \%)$ & $\geq 1(9.1 \%)$ \\
\hline
\end{tabular}

\section{WEIGHT}

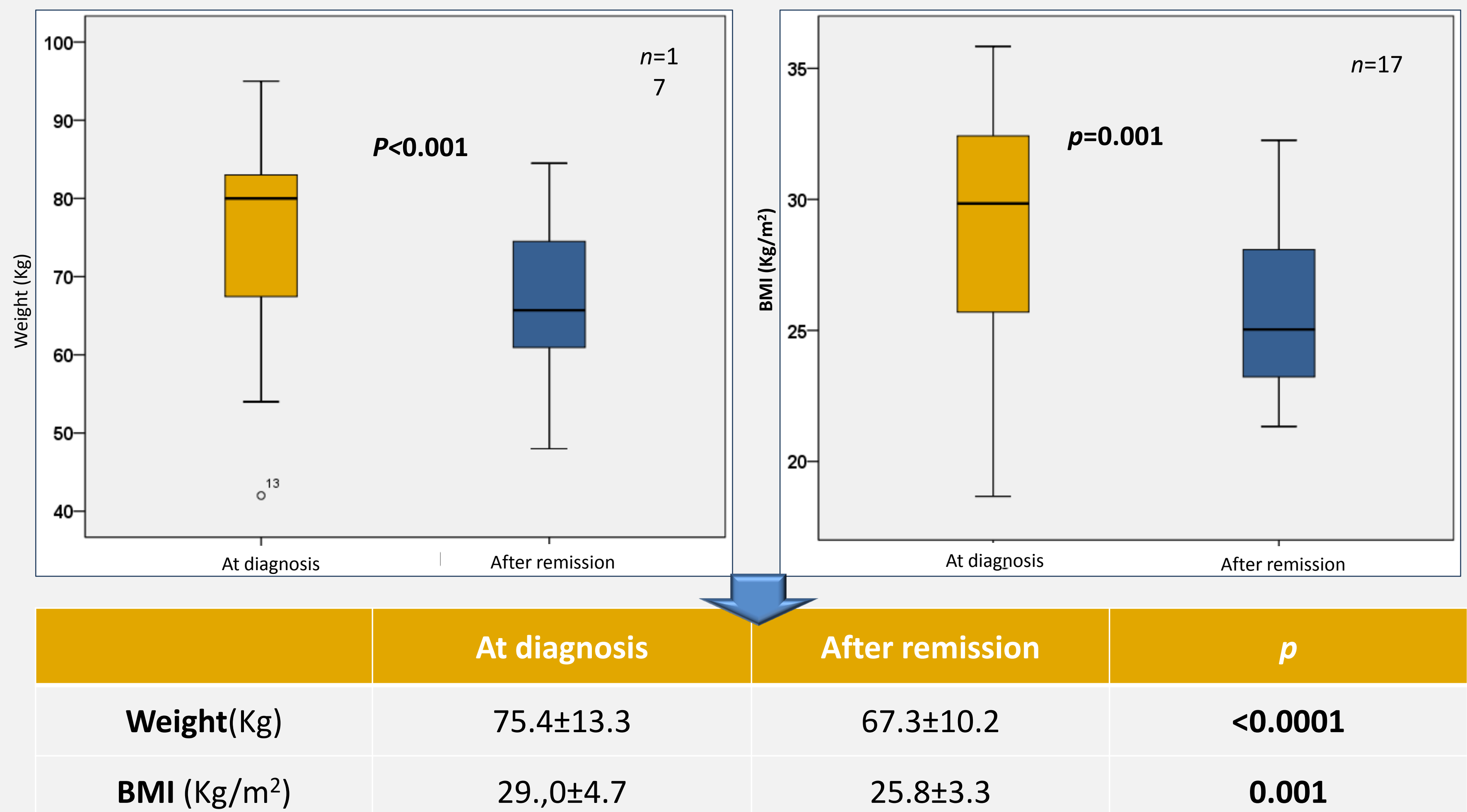

PEDIATRIC PATIENT

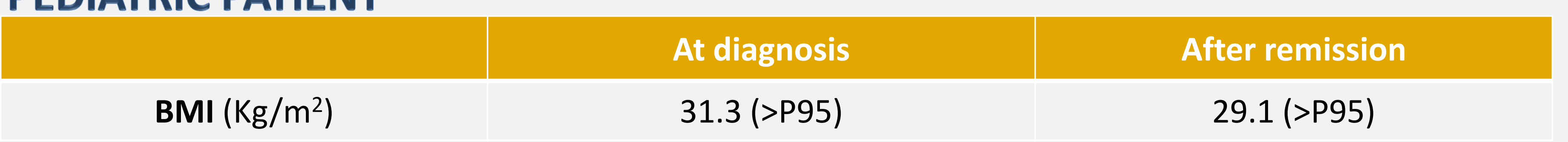

SUMMARY...

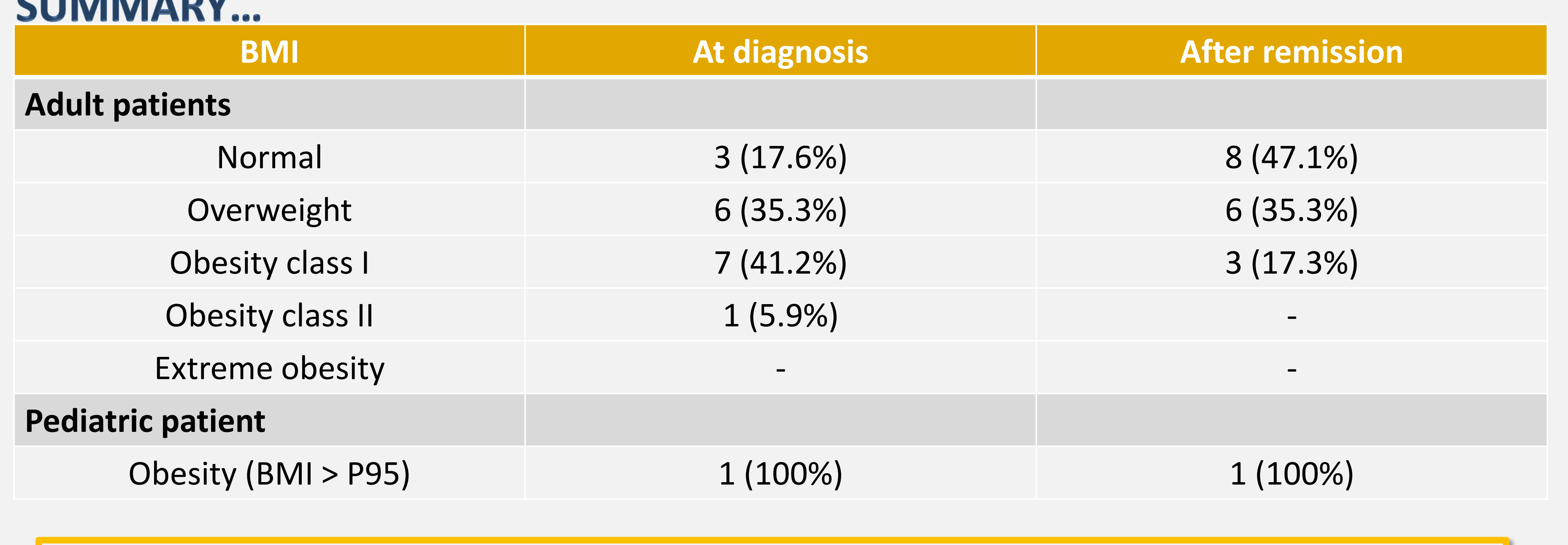

Four patients (22.2\%) remained obese and $6(33.3 \%)$ overweight!

\section{CONCLUSION}

After one year of CD remission, there were significant improvements in hypertension and obesity/overweight. Other comorbidities shown also an improvement, although without statistical significance. Thus, it is crucial to highlight the requirement of maintain surveillance and reassessment of these long-term changes.

\section{REFERENCES}

1. Geer EB et al, Body Composition and Cardiovascular Risk Markers after Remission of Cushing's Diasease: A prospective Study using Whole-Body MRI, J Clin Endocrinol Metab, 2012, 97(5): 1702-1711, Hassan-Smith Lindsay JR et al, The Postoperative Basal Cortisol and TRH Tsphenoidal surgery and pituitary irradiation, UpToDate, 2013;
ACKNOWLEDGEMENTS

To the Portuguese Society of Endocrinology, Diabetes and Metabolism for the financial support to attend the $16^{\text {th }} \mathrm{ECE}$ 